\title{
Lactate Dehydrogenase Increased
}

National Cancer Institute

\section{Source}

National Cancer Institute. Lactate Dehydrogenase Increased. NCI Thesaurus. Code C39595.

A laboratory test result indicating an increase in the lactate dehydrogenase concentration of a biological sample. 